\title{
Arterial Pulsations are Present in One Third of the Human Cranial Vascular Volume Penetrated by Near Infrared Light
}

Stingele, Robert ; Keller, Emanuela ; Wagner, Benedicht P ; Steiner, Thorsten ; Stingele, Karoline ; Hacke, Werner

\begin{abstract}
The percentage of cranial vascular volume that undergoes arterial pulsations is estimated by 18 paired measurements in nine healthy volunteers using near infrared spectroscopy (NIRS, $10 \mathrm{~Hz}$ sampling frequency, 769 $\mathrm{nm}$ ). The NIR absorption is decomposed in pulsatile and non-pulsatile components by digital filtering. The time course of absorption changes of these components after sudden intravenous injection of indocyanine-green (ICG) is used to estimate the arterial fraction of vascular volume (f art ). Approximately $28 \%$ of the vascular volume within the optical field of the NIR spectroscope was arterial. The range of values was $6-43 \%$. Variance of $f$ art was 4.8 times higher between subjects than within subjects, indicating that the variability observed is not due to imprecise estimation but to optode position and subject.
\end{abstract}

DOI: https://doi.org/10.1007/978-1-4615-0075-9_6

Posted at the Zurich Open Repository and Archive, University of Zurich

ZORA URL: https://doi.org/10.5167/uzh-192114

Book Section

Accepted Version

Originally published at:

Stingele, Robert; Keller, Emanuela; Wagner, Benedicht P; Steiner, Thorsten; Stingele, Karoline; Hacke, Werner (2003). Arterial Pulsations are Present in One Third of the Human Cranial Vascular Volume Penetrated by Near Infrared Light. In: Dunn, Jeffrey F; Swartz, Harold M. Oxygen Transport to Tissue XXIV. Cham: Springer, 51-59. DOI: https://doi.org/10.1007/978-1-4615-0075-9_6 
Arterial pulsations are present in one third of the human cranial vascular volume penetrated by near infrared light

Robert Stingele ${ }^{1}$, Emanuela Keller ${ }^{2}$, Benedicht P. Wagner ${ }^{3}$, Thorsten Steiner ${ }^{1}$, Karoline Stingele ${ }^{1} \&$ Werner Hacke ${ }^{1}$

1: Department of Neurology, University of Heidelberg, Germany

2: Department of Neurosurgery, University of Zürich, Switzerland

3: Department of Pediatrics, University of Berne, Switzerland

Corresponding author:

R. Stingele

Department of Neurology

Ruprecht-Karls University

INF 400

D-69120 Heidelberg

Phone: ++49-6221-567504

Email: j62@ix.urz.uni-heidelberg.de 


\section{Abstract:}

The percentage of cranial vascular volume that undergoes arterial pulsations is estimated by 18 paired measurements in nine healthy volunteers using near infrared spectroscopy (NIRS, $10 \mathrm{~Hz}$ sampling frequency, $769 \mathrm{~nm}$ ). The NIR absorption is decomposed in pulsatile and nonpulsatile components by digital filtering. The time course of absorption changes of these components after sudden intravenous injection of indocyanine-green (ICG) is used to estimate the arterial fraction of vascular volume ( $\left.f_{\text {art }}\right)$. Approximately $28 \%$ of the vascular volume within the optical field of the NIR spectroscope was arterial. The range of values was $6-43 \%$. Variance of $\mathrm{f}_{\text {art }}$ was 4.8 times higher between subjects than within subjects, indicating that the variability observed is not due to unprecise estimation but to optode position and subject.

\section{Key words:}

Blood volume; humans; indocyanine-green; NIRS;

\section{Abbreviations:}

$A_{t o t}, A_{a r t}, A_{v e n}, A_{p}:$ NIR absorptions originating in volumes with corresponding indices

$\bar{A}_{p}:$ instantaneous power of pulsatile absorption

$A_{n p}:$ non-pulsatile absorption originating in $V_{v e n}$ and $\bar{V}_{a r t}$

$f_{\text {art }}:$ arterial fraction of arterial vascular volume

ICG: indocyanine-green

RMS: root mean square value

$V_{t o t}, V_{\text {art }}, V_{v e n}, V_{p}:$ Total, arterial, venous, and pulsatile vascular volume

$\bar{V}_{a r t}:$ sum of the cylindrical core volumes of pulsatile vessels

$\bar{V}_{p}:$ instantaneous amplitude of pulsatile volume (= instantaneous root mean square value) 


\section{Introduction:}

The arterial fraction of cranial vascular volume is of interest because oxy- and deoxyhemoglobin, the principal chromophores investigated by NIRS, are present in different concentrations in arterial and venous vessels due to oxygen extraction along the vessel. Therefore, NIRS signals are affected not only by the amount of hemoglobin $(\mathrm{Hb})$ and the overall oxygenation of $\mathrm{Hb}$, but also by $\mathrm{f}_{\text {art }}$ in the optical field at a specific optode position. We present a method allowing non-invasive estimation of $\mathrm{f}_{\text {art }}$ by fast-scanning NIRS and intravenous injection of ICG.

\section{Methods:}

The study was approved by our local ethical committee. Nine healthy volunteers were investigated using NIRS. In each subject, two paired measurements with unchanged optode positions approximately 30 minutes apart were performed. Subjects were breathing spontaneously. Optodes were placed over the lateral aspect of the frontal lobe on either side at a distance of 45 or $55 \mathrm{~mm}$. NIR absorption was recorded at $10 \mathrm{~Hz}$ and $769 \mathrm{~nm}$ using an Oxymon system (University of Nijmegen, Netherlands). After a stable baseline reading was reached, ICG was injected into the cubital vein (ICG Pulsion, total dose 10-30 mg). Both ICG and $\mathrm{Hb}$ are NIR-chromophores with a strict intravascular volume of distribution. NIR absorption due to these chromophores is proportional to their respective intravascular concentrations and the vascular volumes inside the optical field containing the chromophores. The vascular volume in the optical field is subject to periodic changes due to changing transmural blood pressure and therefore NIR absorption is pulsatile. In the present method, pulsatility of blood volume at heart frequency is used as a tag for arterial volume portions. The sampling frequency of $10 \mathrm{~Hz}$ allows to follow pulsatile absorption changes at heart frequency with a large margin of oversampling. To analyze the recorded absorption data, the following model is used. The total volume of blood vessels inside the optical field $\left(\mathrm{V}_{\text {tot }}\right)$ is 
composed of vessels that pulsate at heart frequency $\left(\mathrm{V}_{\text {art }}\right)$ and vessels that do not pulsate in that frequency band $\left(\mathrm{V}_{\mathrm{ven}}\right)$. The arterial volume $\mathrm{V}_{\text {art }}$ is decomposed in two parts. Part one is the sum of the cylindrical core volumes of the arterial vessels $\left(\bar{V}_{a r t}\right)$. This part is constant and it can be pictured as the volume that the arterial vessels would occupy, if there would be no changes in blood pressure. The second part $\left(\mathrm{V}_{\mathrm{p}}\right)$ is the difference between $\bar{V}_{\text {art }}$ and $\mathrm{V}_{\text {art }}$ that is due to changes in transmural blood pressure with the heart cycle. $V_{p}$ is positive in systole, negative in diastole and zero on average. $\mathrm{V}_{\mathrm{p}}$ can be pictured as a cylindrical layer added to the pulsating part of the vessels the thickness of which is changing with blood pressure. In the model used here, the NIR-absorptions $A_{t o t}, A_{v e n}, A_{\text {art }}$, and $A_{p}$ are attributed as originating from the presence of chromophore in the volumes $\mathrm{V}_{\text {tot }}, \mathrm{V}_{\mathrm{ven}}, \mathrm{V}_{\text {art }}$, and $\mathrm{V}_{\mathrm{p}}$. The pulsatile absorption $A_{p}$ is positive in systole, negative in diastole and zero on average, just as the corresponding volume $\mathrm{V}_{\mathrm{p}}$. Before injection of ICG, $\mathrm{A}_{\mathrm{p}}$ is due to presence of $\mathrm{Hb}$ in the arterial part of the vessel and the amplitude of $\mathrm{A}_{\mathrm{p}}$ is constant because the concentration of $\mathrm{Hb}$ is constant. The instantaneous power of the $\mathrm{A}_{\mathrm{p}}$ signal $\left(\bar{A}_{p}\right)$ is constant before ICG appears. When ICG arrives in the optical field for the first time after injection, proportionally filling the two arterial volumes $\left(\mathrm{V}_{\mathrm{p}}\right.$ and $\left.\bar{V}_{a r t}\right)$, the amplitude of $\mathrm{A}_{\mathrm{p}}$ and thus $\bar{A}_{p}$ increase proportional to the increase of the ICG concentration. For convenience non-pulsatile absorption $\left(A_{n p}\right)$ is defined as the lumped absorption originating in non-pulsatile volumes $\bar{V}_{a r t}$ and $V_{v e n}$.

If the concentration of a chromophore is equal in any two compartments in the optical field, then the ratio of the absorptions due to presence of the chromophore in these compartments is equal to the ratio of their volumes. This observation follows from Beer's law because all other factors that determine absorption, such as optical path length, differential path length factor and absorption coefficient simplify when the ratio of absorptions is calculated. Equal concentrations of ICG for two vascular compartments can be assumed in two conditions. Condition 1: In a short period when ICG arrives in the optical field for the first time, the 
concentration of ICG can assumed to be equal in $\mathrm{V}_{\mathrm{p}}$ and $\bar{V}_{\text {art }}$, the two volumes that make up the arterial vessel. During this period of time, the ICG concentration in $\mathrm{V}_{\text {ven }}$ is zero because ICG particles have not yet arrived in the venous volume. The ratio $\bar{A}_{p} / A_{n p}=\bar{A}_{p} /\left(\bar{A}_{a r t}+A_{v e n}\right)=\bar{A}_{p} / \bar{A}_{a r t}$ is equal to the volume ratio $r_{1}=\bar{V}_{p} / \bar{V}_{a r t}$. This ratio indicates the average fraction of the arterial volume that the arterial vessels are inflated and deflated by during the heart cycle.

Condition 2: Many circulation times after the injection of ICG thorough mixing over the entire vascular volume can be assumed and the concentration of ICG is the same in all the volumes mentioned. Calculation of the ratio $\bar{A}_{p} / A_{n p}=\bar{A}_{p} /\left(\bar{A}_{\text {art }}+A_{v e n}\right)=\bar{A}_{p} / \bar{A}_{t o t}$ for this condition yields $r_{2}=\bar{V}_{p} / V_{\text {tot }}$, the ratio indicating the average percentage of volume the arterial vessels are inflated and deflated during the heart cycle in relation to the total vascular volume. Finally, by calculating the quotient

$$
\frac{r_{2}}{r_{1}}=\frac{\bar{V}_{p} / V_{t o t}}{\bar{V}_{p} / \bar{V}_{a r t}}=\frac{\bar{V}_{a r t}}{V_{t o t}}=f_{a r t}
$$

one obtains the desired arterial volume fraction.

The steps of data analysis were as follows:

Digital filters were applied to the raw absorption time series at $769 \mathrm{~nm}$ to obtain $\mathrm{A}_{\mathrm{p}}$ and $\mathrm{A}_{\mathrm{np}}$ $\left(A_{n p}\right.$ : lowpass filter design with edge frequency below heart rate; $A_{p}$ : bandpass filter design with edge frequencies around the heart rate; filters were of Chebyshev type II; the minimum filter order leading to at least $40 \mathrm{~dB}$ of stopband attenuation and less than $0.3 \mathrm{~dB}$ of passband ripple were used).

To obtain an estimate for the time course of the instantaneous power of the $A_{p}$ signal, the signal envelope was calculated (the signal envelope is obtained by taking the absolute value of the analytical signal of $A_{p}$, which in turn is calculated from $A_{p}$ by a Hilbert transform, for details see[1]) (Figure 1). 
To remove the contribution of $\mathrm{Hb}$ to $\mathrm{A}_{\mathrm{np}}$ and the envelope of $\mathrm{A}_{\mathrm{p}}$, the average values of $\mathrm{A}_{\mathrm{np}}$ and of the envelope of $A_{p}$ before injection of ICG were subtracted from the respective time series.

Figure 2 is a parametric plot of the changes in absorption due to ICG only. The abscissa plots non-pulsatile absorption ( $A_{n p}^{I C G}$ ), the ordinate plots the instantaneous RMS amplitude of $\mathrm{A}_{\mathrm{p}}$ $\left(\bar{A}_{p}^{I C G}\right)$, both in optical densities (O.D.). Before injection of ICG, both absorptions are zero. As ICG appears at the arterial extreme of the vessels there is a steep increase of both pulsatile and non-pulsatile absorption. As the first particles of ICG spill over into the non-pulsatile section of the vessels, the non-pulsatile absorption increases relatively more than does the pulsatile absorption. This results in a downward concavity of the initial part of the parametric plot. The initial slope of that section of the parametric plot corresponds to the ratio $r_{1}=\bar{V}_{p} / \bar{V}_{a r t}$. After many circulation times, the concentration of ICG will be equal in the entire vascular volume and thus the amount of ICG entering and leaving the vessels in the optical field during any instant of time will be equal. As ICG is eliminated from circulation the concentration decreases towards zero by the same rate in both pulsatile and non-pulsatile part of the vessels. This results in a linear slope of the parametric plot after about 2 minutes of time have elapsed. The slope of this line corresponds to the ratio $r_{2}=\bar{V}_{p} / V_{t o t}$. As stated above, the quotient of the two slopes gives the desired arterial volume fraction $\mathrm{f}_{\text {art }}$.

\section{$\underline{\text { Results: }}$}

All values reported are means \pm SD. Figure 1 (top) shows the raw absorption data of a representative measurement. The amplitude of pulsatile absorption changes is higher after ICG has been injected (see Figure 1, bottom). The results of filtering the data are shown in Figure 1 (middle and bottom). The envelope of $A_{p}$, shown in black, linearly reflects the concentration of ICG in the pulsatile section of the vessels. 
The maximal change from baseline of the non-pulsatile absorption $A_{n p}$ was $0.25 \pm 0.08$ O.D., the maximal increase of the RMS-amplitude of the pulsatile absorption $\mathrm{A}_{\mathrm{p}}$ was $0.0032 \pm 0.001$ O.D. The parametric plot of RMS-pulsatile versus non-pulsatile absorption changes due to ICG is shown in Figure 2. Both absorptions are zero before ICG is injected. As ICG appears, the parametric plot shows a large increase of both absorptions with initial slope $r_{1}$. The values of $r_{1}$ were $0.022 \pm 0.012$, indicating that the RMS-pulsatile volume change due to blood pressure changes is $2.2 \%$ of the arterial volume $\bar{V}_{a r t}$ on average. As ICG starts to fill nonpulsatile vessels, the parametric plot follows a downward concavity. This can be explained because indicator particles leaving the pulsatile compartment by entering the non-pulsatile compartment will continue to contribute to non-pulsatile absorption but will be lost to detection in the pulsatile compartment. On the other hand, during any instant of time both compartments will be filled by the same number of newly arriving particles. The result is that, in this phase of the parametric plot, the increase of absorption is lower for $A_{p}$ than for $A_{n p}$. After approximately two minutes, both absorptions start to decrease towards zero at the same rate, resulting in a linear slope $r_{2}$ of the parametric plot. The value of $r_{2}$ was $0.0056 \pm 0.0036$ O.D., indicating that the pulsatile RMS-amplitude change was $0.56 \%$ of the total vascular volume. The quotient $r_{2} / r_{1}=f_{\text {art }}$ was $0.28 \pm 0.11$ for the 18 measurements. Therefore, the fraction of arterial volume was about $28 \%$ of the total vascular volume in the optical field. Figure 3 shows the 18 measurement in 9 subjects with paired measurements connected by a line. The range of observed values of $f_{\text {art }}$ was large $(6-43 \%)$. However, the variance within single subjects is smaller than the variance between subjects by a factor of 4.8 (One-way ANOVA for repeated measurements). 


\section{Discussion:}

The arterial fraction of cranial vascular volume $f_{\text {art }}$ is estimated by a non-invasive technique using fast scanning NIRS and intravenous injection of ICG. Knowledge of $\mathrm{f}_{\text {art }}$ is important because the concentration of the principal chromophores investigated by NIRS (oxy- and deoxy- $\mathrm{Hb}$ ) change along the longitudinal axis of the vessels due to desaturation. With unchanged oxygenation of hemoglobin, a change of optode position to an optical field with higher $\mathrm{f}_{\text {art }}$ will give higher contributions of oxy-Hb and lower contributions of deoxy-Hb. The method is based on the decomposition in pulsatile and non-pulsatile components of NIRS absorption data collected before and during the passage of ICG through the vascular bed under the NIRS-detector. The method relies on the following assumptions.

1) The concentration of ICG becomes equal in all vascular sections in the optical field after many circulation times. This assumption is certainly reasonable, but it remains to be defined what exactly 'many circulation times' means. A strength of the present method is that it provides an indication of the time after which thorough mixing of ICG has occurred. The point of the parametric plot after which both pulsatile and non-pulsatile absorptions decrease towards zero at the same rate indicates that the outflow of ICG from both compartments is identical during any instant of time. Since the compartments share the same ICG influx, this statement is identical with saying that the concentration of ICG is identical in the compartments. In the 18 measurements, identical concentrations in the compartments were reached approximately 2 minutes after injection of ICG.

2) The concentration in the non-pulsatile part of the vessels is zero when the indicator starts to arrive in the optical field. This assumption is true only, if the vascular system inside the optical field is pictured by a single vessel, in which sequential filling of arterial and venous compartments is guaranteed. The assumption may be in error in biological tissues, in which the optical field is perfused by a large number of vessels. Some of these vessels might have their pulsatile components outside the optical field, therefore not contributing to $A_{p}$, but their 
non-pulsatile components inside the optical field, thereby contributing to $\mathrm{A}_{\mathrm{np}}$. This anatomical characteristic of an unknown portion of $\mathrm{V}_{\text {tot }}$ per se does not influence the accuracy of the measurement. However, if this portion of vessels happens to be filled sooner after injection of ICG than the remaining vessels, a systematic error of measurement results. More precisely, the initial slope $\left(r_{1}\right)$ of Figure 2 becomes false low because initial non-pulsatile absorption results not only from the core volumes $\mathrm{V}_{\text {art }}$ of the arterial vessels but also from the earlyfilling venous vessels that do not contribute to $A_{p}$. Thus, overestimation of the arterial volume fraction $\mathrm{r}_{2} / \mathrm{r}_{1}$ is the result. This effect might lead to serious errors when a large portion of $\mathrm{V}_{\text {tot }}$ is made up from vessels that have a large percentage of their pulsatile parts outside the optical field and if these vessels are filled early, for example in arterio-venous shunting. The error of measurement introduced by this effect in physiological situations cannot be estimated at the moment, but we think that it is small because both the anatomic peculiarity of the vessel and early filling have to coincide in order to affect the measurement.

3) The concentration of ICG is equal in $\mathrm{V}_{\mathrm{p}}$ and $\bar{V}_{a r t}$. This statement is equivalent to the assumption of complete radial mixing in the arterial vessels. Uniform concentration of the indicator at any point of the cross-section of a vessel is established faster than mixing along the longitudinal axis because the distances the indicator has to travel are shorter. In the present case, due to intravenous injection of ICG, the time elapsed until ICG appears in the optical field is on the order of 10-15 seconds. During this time ICG has to cross the capillary bed of pulmonary circulation. Therefore, thorough radial mixing seems to be a safe assumption in this case.

A potential source of error inherent to this method is the determination of the initial slope $r_{1}$ of the parametric plot. While $\mathrm{r}_{2}$ can be estimated accurately due to the large number of data points collected during elimination of ICG over several minutes, the value of $r_{1}$ relies on a few data points only (Figure 2). The reason for this is that ICG does not appear in the optical field as a step concentration increase. The leading edge of the ICG front approaching, made up of 
those ICG particles with the shortest transit times from the site of injection to the optical field has a lower ICG concentration than the bulk of indicator arriving a little later. Therefore, during the initial phase of the parametric plot used to measure $r_{1}$, the concentration of ICG is low in the arterial vessels. This could be improved on by a closer site of injection for ICG (e.g. central venous catheter) leading to a more compact ICG bolus. Furthermore, the error introduced by this effect is not systematic, because $r_{1}$ can be equally over- and underestimated. Therefore, averaging more than one measurement of $r_{1}$ should improve precision.

\section{$\underline{\text { References: }}$}

1. Oppenheim AV, Schafer RW. Discrete-Time Signal Processing. In: Prentice Hall Signal Processing Series $1^{\text {st }}$ ed. Englewood Cliffs: Prentice Hall, 1989.

\section{Figure legends:}

Figure 1: Raw NIR absorption data at $769 \mathrm{~nm}$ sampled at $10 \mathrm{~Hz}$ (top). Lowpass filtered data (middle), and bandpass filtered data (bottom, in gray). The black line overlaid to the bottom curve is the envelope of the bandpass filtered data. Note the different scale of the ordinate in the bottom panel. 


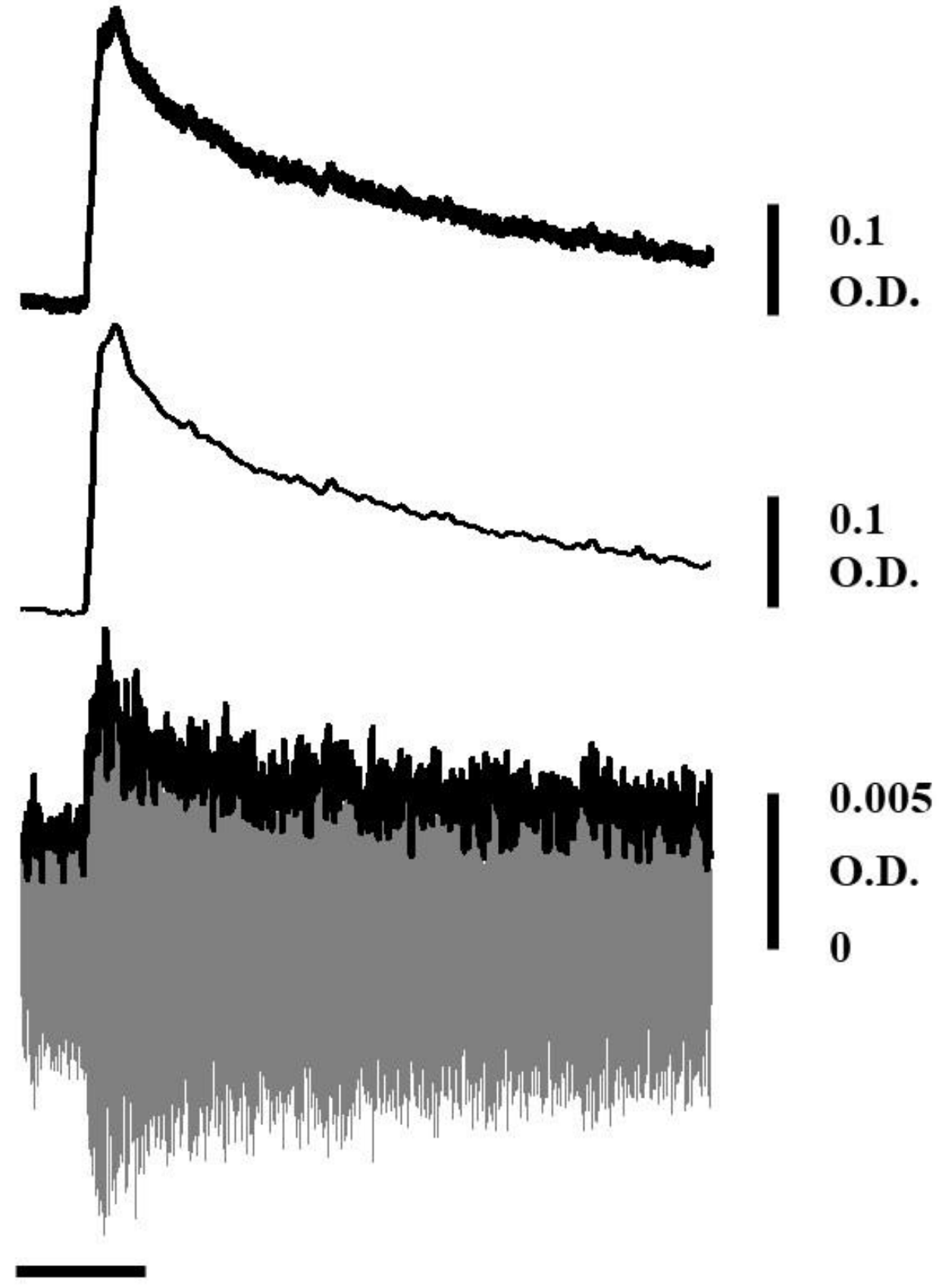

$100 \mathrm{sec}$ 
Figure 2: Parametric plot of pulsatile (ordinate) versus non-pulsatile (abscissa) absorption after injection of ICG. Small data points are $0.1 \mathrm{sec}$ apart. The large circles are $10 \mathrm{sec}$ apart. For explanation of the slopes $r_{1}$ and $r_{2}$ see text. 


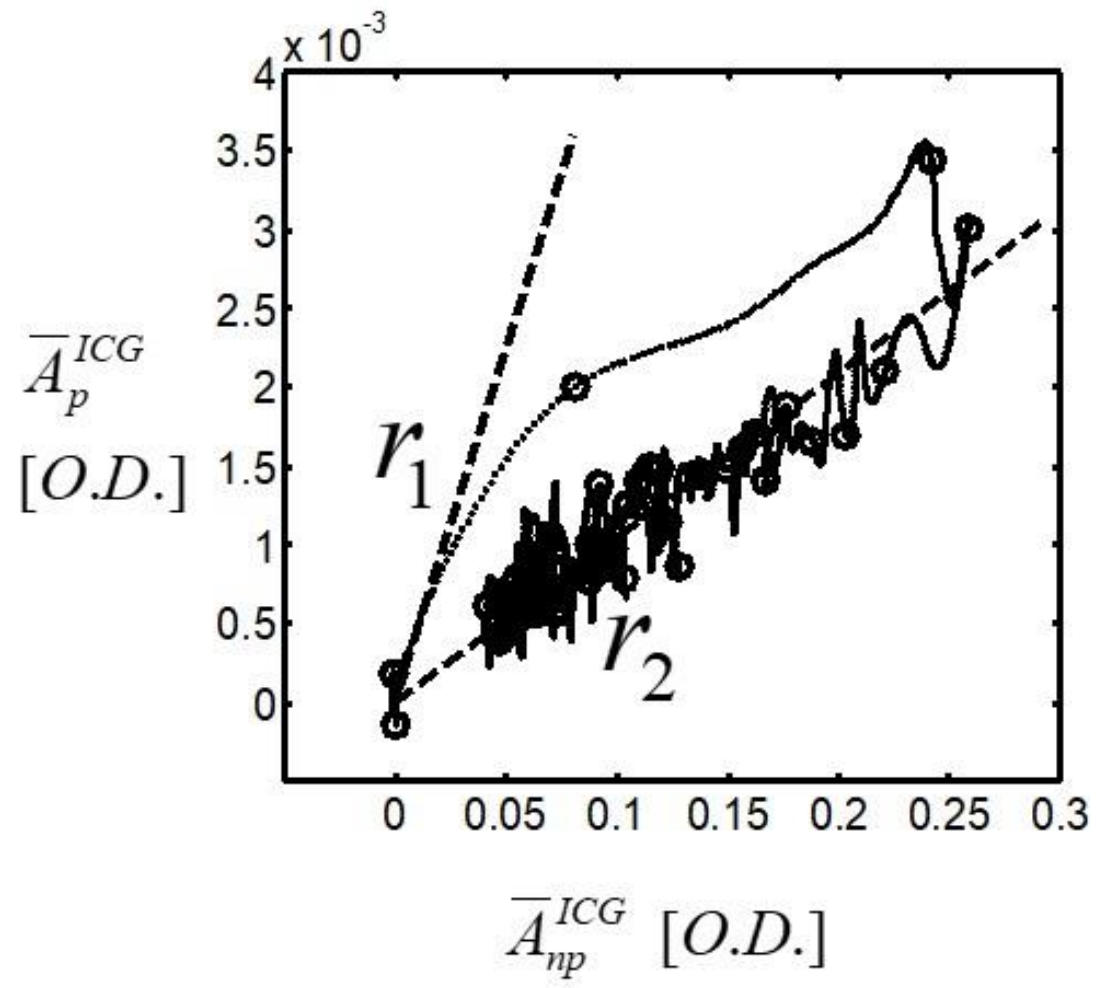


Figure 3: Arterial fraction of blood volume in percent. 18 measurements were performed in 9 subjects. Paired measurements are connected by a line. Mean $\mathrm{f}_{\text {art }}$ was $28 \%$. There is a large range of observed values (6-24\%). Variability between subjects is larger than variability within single subjects by a factor of 4.8 (One-way ANOVA for repeated measurements). 


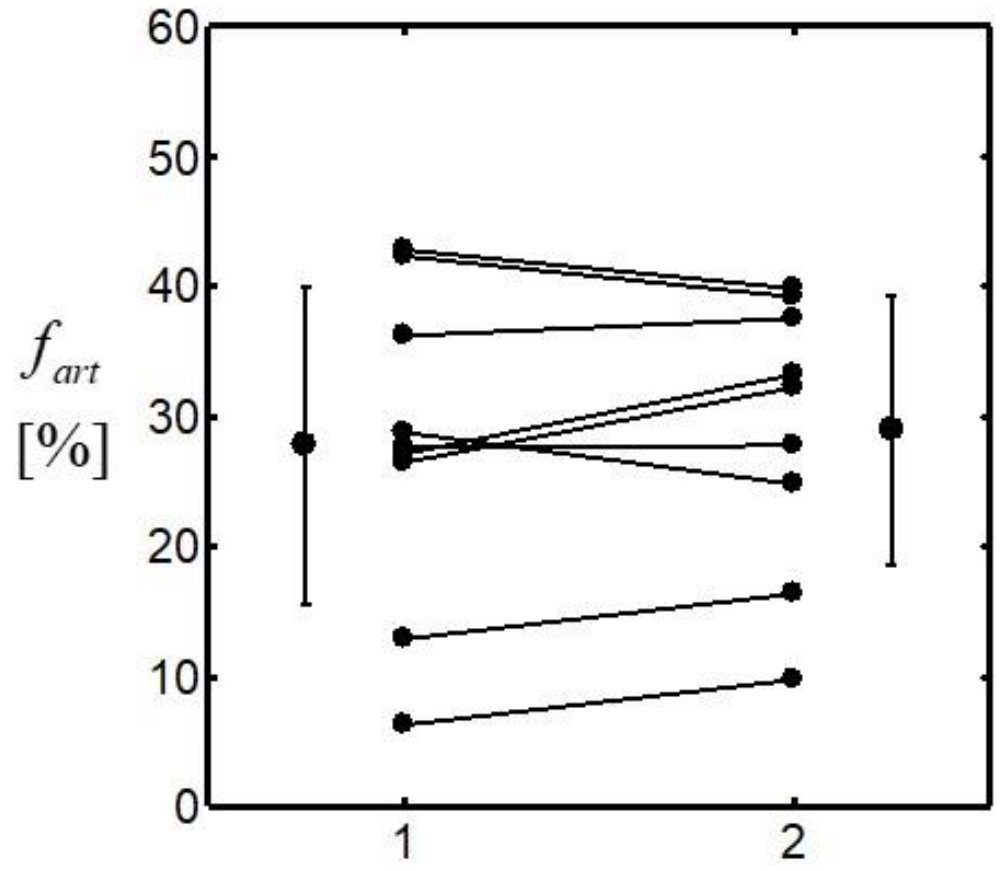

Measurement 